\title{
Immunological profile of resistance and susceptibility in naturally infected dogs by Leishmania infantum
}

\author{
Gleisiane Gomes de Almeida Leal ${ }^{\mathrm{a}}$, Bruno Mendes Roatt ${ }^{\mathrm{a}, \mathrm{b}, \mathrm{f}}$, \\ Rodrigo Dian de Oliveira Aguiar-Soares ${ }^{\mathrm{a}, \mathrm{b}}$, Cláudia Martins Carneiro ${ }^{\mathrm{a}, \mathrm{b}}$, \\ Rodolfo Cordeiro Giunchetti ${ }^{\mathrm{a}, \mathrm{c}}$, Andréa Teixeira-Carvalho ${ }^{\mathrm{d}}$, \\ Olindo Assis Martins-Filho ${ }^{\mathrm{d}}$, Amanda Fortes Francisco ${ }^{\mathrm{a}}$, \\ Jamille Mirelle Cardoso $^{a}$, Fernando Augusto Siqueira Mathias ${ }^{a}$, \\ Rodrigo Correa-Oliveira ${ }^{e, f}$, Mariângela Carneiro ${ }^{g}$, Wendel Coura-Vital ${ }^{\text {b,f,g, }}$, \\ Alexandre Barbosa Reis ${ }^{a, b, f, *}$
}

\footnotetext{
a Laboratório de Imunopatologia, Núcleo de Pesquisas em Ciências Biológicas/NUPEB, Universidade Federal de Ouro Preto, Ouro Preto, Minas Gerais, Brazil

b Laboratório de Pesquisas Clínicas, Departamento de Análises Clínicas, Escola de Farmácia, Universidade Federal de Ouro Preto, Ouro Preto, Minas Gerais, Brazil

c Laboratório de Biologia das Interações Celulares, Departamento de Morfologia, Universidade Federal de Minas Gerais, Belo Horizonte, Minas Gerais, Brazil

d Laboratório de Biomarcadores de Diagnóstico e Monitoração, Centro de Pesquisas René Rachou, Fundação Oswaldo Cruz-FIOCRUZ, Belo Horizonte, Minas Gerais, Brazil

e Laboratório de Imunologia Celular e Molecular, Centro René Rachou, Fundação Oswaldo Cruz, Belo Horizonte, Minas Gerais, Brazil

f Instituto Nacional de Ciência e Tecnologia em Doenças Tropicais, INCT-DT, Brazil

g Pós-Graduação em Infectologia e Medicina Tropical, Faculdade de Medicina, Universidade Federal de Minas Gerais, Belo Horizonte,

Minas Gerais, Brazil
}

\section{A R T I C L E I N F O}

\section{Article history:}

Received 23 January 2014

Received in revised form 21 August 2014

Accepted 25 August 2014

\section{Keywords:}

Canine visceral leishmaniasis

Leishmania infantum

Clinical forms

Immunophenotyping

Cytokines

\begin{abstract}
A B S T R A C T
Visceral leishmaniasis has a great impact on public health, and dogs are considered the main domestic reservoir of Leishmania infantum, the causal parasite. In this study, 159 animals naturally infected by L. infantum from an endemic area of Brazil were evaluated through an analysis of cellular responses, using flow cytometry, and of the hematological parameters. The results confirmed that disease progression is associated with anemia and reductions in eosinophils, monocytes and lymphocytes. The investigation of the immune response, based on the immunophenotypic profile of peripheral blood, showed declines in the absolute numbers of $\mathrm{T}$ lymphocytes $\mathrm{CD} 5^{+}$and their subsets $\left(\mathrm{CD} 4^{+}\right.$and $\left.\mathrm{CD} 8^{+}\right)$and a drop of $\mathrm{B}$ lymphocytes in asymptomatic seropositive (AD-II) and symptomatic seropositive (SD) dogs. Neutrophils, when stimulated with soluble antigen of L. infantum, showed higher synthesis of interferon (IFN)- $\gamma^{+}$in AD-II and SD groups, with decreased production of interleukin
\end{abstract}

\footnotetext{
* Corresponding author at: Laboratório de Imunopatologia, Núcleo de Pesquisas em Ciências Biológicas, ICEB II, Morro do Cruzeiro, Universidade Federal de Ouro Preto, Ouro Preto, MG CEP 35400-000, Brazil. Tel.: +55 2131 3559 1694; fax: +55 213135591680 .

E-mail addresses: alexreisufop@gmail.com, alexreis@nupeb.ufop.br (A.B. Reis).
} 
(IL) $-4^{+}$in asymptomatic seronegative dogs positive for $L$. infantum infection based on polymerase chain reaction testing (AD-I group). In the AD-II and SD groups, subpopulations of stimulated lymphocytes $\left(\mathrm{CD} 4^{+}\right.$and $\left.\mathrm{CD}^{+}\right)$also exhibited greater synthesis of IFN- $\gamma^{+}$and IL- $4^{+}$in culture. These results suggest that the animals of the AD-II and SD groups exhibited a mixed immune response (Type 1 and 2 ) and the AD-I group presenting an immune profile very similar to normal control animals.

(c) 2014 Elsevier B.V. All rights reserved.

\section{Introduction}

Visceral leishmaniasis (VL) caused by the protozoan Leishmania (Leishmania) infantum, is one of the most important zoonotic diseases affecting dogs and humans in South and Central America, the Mediterranean basin and parts of Asia (World Health Organization, 2010). Dogs (Canis familiaris) are the most important reservoir of the parasite in urban areas, especially those that have a high parasite burden in the skin and a high prevalence in this environment (Giunchetti et al., 2006; Coura-Vital et al., 2011b). It has often been observed that an increase in canine visceral leishmaniasis (CVL) cases precedes a rise in human cases (Fraga et al., 2012; Grimaldi et al., 2012).

CVL may evolve from a nonapparent infection to a severe and systemic disease, which usually culminates in death. Asymptomatic dogs can recover or develop clinical symptomatic disease, or they may remain infected for years, even lifelong, without clinical manifestation (Reis et al., 2009). Recently it has been shown that a high percentage of asymptomatic infected dogs are PCR positive but seronegative (Coura-Vital et al., 2011b). These animals, although their infection status is not detected by conventional serology, are more likely to seroconvert (Coura-Vital et al., 2013). They also apparently have a different type of immune response that seems to be related to resistance and is characterized by high proportions of $\mathrm{CD} 4^{+} \mathrm{T}$ lymphocytes and $\mathrm{CD} 21^{+} \mathrm{B}$ cells and high expression of IFN- $\gamma$ (Reis et al., 2006b; Coura-Vital et al., 2011a; Menezes-Souza et al., 2011).

The components of innate and adaptive immunity engage in a range of interactions that is remarkably diverse and complex (Reis et al., 2009, 2010). The course of CVL is interconnected with the host immune response and the persistence and proliferation of the parasites throughout the skin and visceral organs. The innate immune response has a relevant role in protecting against the parasite in addition to switching on the adaptive response that can control the Leishmania infection without the development of a specific adaptive immunity (Moreno and Alvar, 2002). Studies indicate that the successful resolution of Leishmania infections depends on the ability of the host to mount a specific T-cell response, with the activation of macrophages mediated by cytokines derived from $\mathrm{T}$ cells (Carrillo and Moreno, 2009). Symptomatic dogs that develop severe disease already exhibit clear suppression of specific types of cell-mediated immunity, particularly $\mathrm{CD}^{+} \mathrm{T}$ lymphocytes (Pinelli et al., 1994). Resistance to infection is associated with a Type 1 response, with a predominance of IL-12,
IFN- $\gamma$, IL- 2 and tumor necrosis factor (TNF)- $\alpha$, which will increase the efficiency of phagocytic cells and cytotoxic lymphocytes, triggering a protective immune response. On the other hand, susceptibility to infection is associated with a Type 2 response, with predominance of IL-4, IL-5, IL-10, IL-13 and TGF- $\beta$ (Pinelli et al., 1995, 1999a; Correa et al., 2007; Lage et al., 2007; Menezes-Souza et al., 2011).

In a previously reported study, our group showed that asymptomatic dogs (seronegative/PCR+ [AD-I] and seropositive [AD-II]) appear to have a dichotomous infection spectrum that influences the humoral and cellular immunological status in CVL (Coura-Vital et al., 2011a). The aim of the present study was to investigate immunological events in naturally infected dogs based on the dichotomy between asymptomatic groups (AD-I and AD-II) and to identify biomarkers associated with resistance and susceptibility to infection by L. infantum.

\section{Materials and methods}

\subsection{Experimental design}

The present study included 159 mongrel dogs of both sexes (81 male and 78 female) from an endemic area of Brazil. The mean age was 49.8 months (SD 37.8), and the median was 42 months (IQR 24; 66). The samples were collected from domestic dogs at the Zoonoses Control Centre of Belo Horizonte. Serological tests (immunofluorescence antibody test [IFAT] and ELISA) were performed following the manufacturer's instructions. Dogs with an IFAT titer $<1 / 40$ were considered seronegative, and dogs with IFAT titer $\geq 1 / 40$ were considered seropositive and infected with Leishmania spp. The serological tests were performed in the Laboratory of Zoonosis at the Belo Horizonte Health Department. Because of the high prevalence of seronegative/PCR+ dogs in the endemic area (Coura-Vital et al., 2011b), the seronegative dogs were subjected to molecular testing. Molecular testing (PCR) was performed in buffy coat samples with primers from a conserved region of the Leishmania kDNA minicircle (P150-152) (Passos et al., 1999). A single PCR product of $120 \mathrm{bp}$ was generated (Degrave et al., 1994). The reaction mixture was performed as described by Coura-Vital et al. (2011b), and the species of Leishmania was determined by RFLP-PCR (Volpini et al., 2004). In this study, the dogs were not submitted to tests against other canine vector-borne diseases. 


\subsection{Serological assays}

The ELISA was performed using the EIE-LVC ${ }^{\circledR}$ kit (BioManguinhos/Fiocruz, Rio de Janeiro, Brazil) according to the manufacturer's instructions. The reactions were performed and samples with optical density above the cut-off were considered positive. The cut-off was defined on each plate by considering the mean of the optical density of the negative controls multiplied by two. The assays were read on an automatic EL 800G ELISA microplate reader.

The IFAT was conducted using the IFI-LVC ${ }^{\circledR}$ kit (BioManguinhos/Fiocruz, Rio de Janeiro, Brazil). The tests were executed according to the manufacturer's instruction. The slides were prepared and examined using a fluorescent microscope with $40 \times$ objective (Olympus BX40). The results were considered positive when the fluorescent parasites were observed at serum titer of 1:40 or more.

\subsection{Clinical groups}

Dogs were clinically classified according to serological (ELISA and IFAT) and molecular tests (PCR-RFLP) and by clinical features, as described by Coura-Vital et al. (2011a), and were divided into four groups. Dogs with no clinical signs and negative serological and molecular results composed the control group (CD; $n=44)$. Seronegative dogs without clinical signs but positive molecular results for $L$. infantum were classified as asymptomatic dogs I (AD-I; $n=53$ ). Dogs with positive serological results for Leishmania spp. but no clinical signs were classified as asymptomatic dogs II (AD-II; $n=20$ ). Dogs with clinical signs and positive serological results were classified as symptomatic dogs ( $\mathrm{SD} ; n=38)$. With regard to gender, mean age (in months) and standard deviation these groups have the following characteristics: CD $\left(27 \circ^{7}\right.$ and 17\%; [52.4; SD 38.2]); AD-I (24ه and 29\%; [51.0; SD 38.4]); AD-II (9॰ and 11\%; [45.4; SD 17.7]) and SD (20 $\circ^{\circ}$ and 18\%; [45.5; SD 22.8]).

For immunophenotyping and intracytoplasmic cytokine assays, 124 of the 159 dogs were grouped as follows: $\mathrm{CD}(n=28), \mathrm{AD}-\mathrm{I}(n=34), \mathrm{AD}-\mathrm{II}(n=20)$ and $\mathrm{SD}(n=42)$. Already these groups the gender age of the animals and standard deviation were: $\mathrm{CD}\left(18 \sigma^{\circ}\right.$ and 10 ; [50.6; SD 39.5]); AD-I (17॰ and 17\%; [50.9; SD 35.8]); AD-II

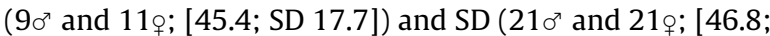
SD 22.6]).

\subsection{Blood samples and hematological evaluation}

Peripheral blood ( $5 \mathrm{~mL}$ ) from the brachiocephalic or jugular vein was collected into tubes containing ethylene diamine tetracetic acid (EDTA) at a final concentration of $1 \mathrm{mg} / \mathrm{mL}$ for the hemogram, blood film and immunological evaluation by immunophenotyping (ex vivo) by flow cytometry. Erythrocytes and leukocytes were quantified using an automatic cell counter (Model 2800 Vet, Mindray). Morphological characteristics of the blood cells and differential leukocyte counts were obtained by blood smear analysis after prior staining by routine methods. To conduct the tests for evaluating the immune response after in vitro stimulation, $5 \mathrm{~mL}$ of a heparinized peripheral blood sample from each dog was transferred to sterile heparinized tubes (BD Pharmingen, San Diego, CA).

\subsection{Canine blood leukocyte immunophenotyping}

Immunophenotyping of peripheral blood by flow cytometry was carried out using simple markup of five primary 5-mL polystyrene tubes (Falcon ${ }^{\circledR} 2054$, Becton Dickinson, San Diego, USA) with the following mAbs: diluted phycoerythrin (PE)-labeled anti-canine CD5 (1:400, mouse IgG2a, clone YKIX322. 3), anti-canine CD4 (1:1000, mouse IgG2a, clone YKIX302. 9) and diluted fluorescein isothiocyanate (FITC) anti-canine CD8 (1:80, mouse IgG1, clone YCATE55. 9)(Serotec, USA). The mAbs were used in an indirect immunofluorescence procedure in which pooled normal rat serum (diluted 1:6000) was used as the isotypic control. A mouse anti-human CD21 PE (1:100, mouse IgG1, clone IOBla; Immunotech Co. Marseille, France) and a diluted PE/Cy-5 conjugated mouse anti-human CD14 (1:300 mL, 1:200, IgG2a, clone TÜK4; Serotec, USA) were also used. Fifty microliters of blood collected in EDTA was added to tubes containing $50 \mu \mathrm{L}$ of antibody and incubated for $30 \mathrm{~min}$ at room temperature (RT). Afterwards, the erythrocytes were lysed by adding $2 \mathrm{~mL}$ of lysis solution (FACS brand lysing solution; Becton Dickinson, San Diego, CA, USA), which was followed by incubation for $10 \mathrm{~min}$ at RT. The leukocytes were then washed twice with $2 \mathrm{~mL}$ of PBS (phosphate buffered saline $0.15 \mathrm{M}, \mathrm{pH} 7.2$ ) and centrifuged at $400 \times g$ for $10 \mathrm{~min}$ at RT. Then, the labeled cells were fixed for $30 \mathrm{~min}$ at RT with $200 \mu \mathrm{L}$ of FACS FIX solution ( $10 \mathrm{~g} / \mathrm{L}$ paraformaldehyde; $10.2 \mathrm{~g} / \mathrm{L}$ sodium cacodylate and $6.65 \mathrm{~g} / \mathrm{L}$ sodium chloride, $\mathrm{pH} 7.2$ ). Flow cytometric measurements were performed on a FACScalibur ${ }^{\mathrm{TM}}$ instrument (Becton Dickinson, Mountain View, CA), and the data were analyzed using FlowJo ${ }^{\circledR}$ software $(20,000$ events acquired per sample). The results were expressed in absolute counts (cell number $/ \mathrm{mm}^{3}$ ) through the product of the percentage of positive cells $\left(\mathrm{CD}^{+}, \mathrm{CD}^{+}, \mathrm{CD}^{+}\right.$and $\mathrm{CD} 21^{+}$) within gated lymphocytes by absolute lymphocyte counts. The absolute counts for monocytes were obtained through the products of CD14 ${ }^{+}$cells within ungated leukocytes by the selection of the region of interest, based on morphometric and immunophenotypic graphics of distribution CD14/FL3 versus SSC, to identify the population of $\mathrm{CD} 14^{+ \text {High }}$ and SSC ${ }^{\text {Intermediate }}$, minimizing the contamination of this population with lymphocytes and neutrophils.

\subsection{Antigen production for in vitro assays}

Soluble L. infantum (MHOM/BR/1070/BH46) antigen (SLAi) was prepared as described by Reis et al. (2006c) from promastigotes harvested from stationary-phase in liver infusion tryptose cultures. The concentration of protein in the SLAi solution was determined as previously described Lowry et al. (1951) and adjusted to $1 \mathrm{mg} / \mathrm{mL}$. Diluted SLAi was divided into small portions and stored at $-80^{\circ} \mathrm{C}$ until required for assays. 
Table 1

Hematological parameters of dogs naturally infected with Leishmania infantum and uninfected dogs.

\begin{tabular}{|c|c|c|c|c|}
\hline \multirow[t]{2}{*}{ Hematological parameters } & \multicolumn{4}{|l|}{ Clinical groups } \\
\hline & $\mathrm{CD}$ & AD-I & AD-II & SD \\
\hline Erythrocytes $\left(10^{6} / \mathrm{mm}^{3}\right)$ & $7.3(6.8-8.2)$ & $7.41(6.6-7.9)$ & $6.2(5.3-6.5)^{\mathrm{a}, \mathrm{b}}$ & $4.6(3.9-5.5)^{a, b}$ \\
\hline Hemoglobin (g\%) & $15.0(13.6-16.2)$ & $15.6(13.8-17.1)$ & $13.0(11.5-13.9)^{a, b}$ & $9.4(7.5-11.5)^{\mathrm{a}, \mathrm{b}, \mathrm{c}}$ \\
\hline Hematocrit (\%) & $40.8(37.0-45.0)$ & $41.4(39.0-46.3)$ & $40.9(34.5-44.9)$ & $29.8(24.6-34.1)^{a, b, c}$ \\
\hline Platelets $\left(10^{3} / \mathrm{mm}^{3}\right)$ & $222.0(159.0-321.0)$ & $258.0(171.0-342.0)$ & $199(148.0-240.0)$ & $202.5(114.8-250.0)^{\mathrm{b}}$ \\
\hline Leucocytes $\left(10^{3} / \mathrm{mm}^{3}\right)$ & $11.2(9.0-13.4)$ & $11.1(9.6-14.1)$ & $8.4(7.6-11.3)^{\mathrm{b}}$ & $10.8(7.2-12.8)$ \\
\hline Total neutrophils & $5.2(4.3-7.0)$ & $5.8(4.5-7.3)$ & $5.4(4.3-7.9)$ & $7.0(4.2-9.6)$ \\
\hline Eosinophils & $0.5(0.4-0.7)$ & $0.4(0.3-0.7)$ & $0.3(0.2-0.5)^{\mathrm{a}, \mathrm{b}}$ & $0.3(0.1-0.4)^{a, b}$ \\
\hline Monocytes & $0.5(0.4-0.7)$ & $0.5(0.4-0.6)$ & $0.3(0.2-0.4)^{a, b}$ & $0.3(0.2-0.4)^{\mathrm{a}, \mathrm{b}}$ \\
\hline Lymphocytes & $4.3(3.6-5.5)$ & $4.4(3.6-5.5)$ & $2.7(2.4-3.7)^{\mathrm{a}, \mathrm{b}}$ & $2.5(2.0-3.4)^{\mathrm{a}, \mathrm{b}}$ \\
\hline
\end{tabular}

Results are shown as median values and the brackets first (Q1) and third (Q3) quartile.

$\mathrm{CD}$, control dogs; AD-I, asymptomatic dogs I; AD-II, asymptomatic dogs II; SD, symptomatic dogs.

a Statistically significant differences compared with CD.

b Statistically significant differences compared with AD-I.

c Statistically significant differences compared with AD-II.

\subsection{Immunostaining for cell surface markers and intracellular cytokines}

Blood samples were collected in sterile tubes containing sodium heparin at a final volume of $5 \mathrm{~mL}$ of peripheral blood. Monoclonal antibodies used to detect cell surface markers included anti-canine FITC-CD4 antibody (1:200, mouse IgG2a, clone YKIX322.3) and anti-canine FITC-CD8 antibody (1:100, mouse IgG2a, clone YKIX302 9). Additionally, mAbs cross-reactive with canine cytokines were used for intracytoplasmic staining, including anti-bovine PE-IFN- $\gamma$ antibody (clone CC302) and anti-bovine PE-IL4 antibody (clone CC303), all purchased from Serotec (Oxford, UK).

Two 14-mL polypropylene tubes were prepared for each animal studied; one served as a control tube ( $1 \mathrm{~mL}$ RPMI plus $1 \mathrm{~mL}$ of whole blood in heparin), and in the stimulated tube $L$. infantum antigen was added at final concentration of $25 \mu \mathrm{g} / \mathrm{mL}$. The tubes were incubated for $12 \mathrm{~h}$ and kept at $37^{\circ} \mathrm{C}$ in an incubator with $5 \% \mathrm{CO}_{2}$. Brefeldin A-BFA (Sigma, St Louis, MO, USA) was added to each tube at a final concentration of $10 \mu \mathrm{g} / \mathrm{mL}$, and cultures were then submitted to an additional $4 \mathrm{~h}$ of incubation in $5 \% \mathrm{CO}_{2}$ humidified incubator at $37^{\circ} \mathrm{C}$. A tube containing PMA at a final concentration of $25 \mathrm{ng} / \mathrm{mL}$ was used as a positive control at final $4 \mathrm{~h}$ of incubation as BFA. First staining was performed for monoclonal anti-surface molecules (CD4 ${ }^{+}$and $\mathrm{CD}^{+}$). After resuspension of these labeled cells, we proceeded to stain intracytoplasmic cytokines (anti-IFN- $\gamma$ and anti-IL-4) in U-bottom 96-well plates. The microtubes were kept at $4{ }^{\circ} \mathrm{C}$ until the acquisition of counts on the flow cytometer (FACScalibur - Becton Dickinson, San Jose, CA, USA), which evaluated at least 30,000 events per tube.

Distinct gating strategies were used to select the leukocyte subpopulations. The canine neutrophils were identified and selected based on their unique expression of CD4 cell surface marker, using side scatter (SSC) versus FL1/anti-CD4 FITC dot plot distributions, thus minimizing contamination of the selected region by monocytes and eosinophils. The eosinophils were identified and selected based on their autofluorescence, using nonrelated FL-3 channel versus forward scatter (FSC) dot plot distributions. The analysis of the cytokine profile of $\mathrm{CD} 4^{+}$and $\mathrm{CD} 8^{+}$
T-cell subsets was performed by first establishing a scattering gate on the lymphocyte population, using laser SSC versus FL1 dot plot distributions. After selecting the region of interest (R1) dot plots were constructed for FSC versus IFN- $\gamma /$ FL2 or IL-4/FL2 to determine the percentage of IFN$\gamma^{+}$or IL- $4^{+}$cells within the population of neutrophils and eosinophils previously selected in $\mathrm{R} 1$. To evaluate the $\mathrm{CD} 4^{+}$ and $\mathrm{CD}^{+} \mathrm{T}$ subsets, dot plots were used for CD4/FL1 or CD8/FL1 versus IFN- $\gamma /$ FL2 or IL-4/FL2. The results were expressed in percentage of cells (IFN- $\gamma^{+}$neutrophils, IL- $4^{+}$ neutrophils, IFN- $\gamma^{+}$eosinophils, IL- $4^{+}$eosinophils, IFN- $\gamma^{+}$ $\mathrm{CD} 4^{+}, \mathrm{IL}_{-} 4^{+} \mathrm{CD} 4^{+}, \mathrm{IFN}-\gamma^{+} \mathrm{CD} 8^{+}$and $\mathrm{IL}-4^{+} \mathrm{CD} 8^{+}$).

\subsection{Statistical analysis}

Statistical analysis was performed using GraphPad Prism 5.0. The normality of the data was assessed using the Kolmogorov-Smirnoff test. Considering the nonparametric nature of all data sets, Kruskal-Wallis tests were used to investigate differences between the groups, followed by Dunn's test for pairwise comparisons. Spearman's rank correlation was computed to investigate associations between cell immunophenotyping and cytokineproducing cells and the clinical groups. The Chi-square test and Kruskal-Wallis test were used to evaluate if the clinical groups were homogeneous in relation to gender and age respectively. Significant differences were considered at $p<0.05$.

\subsection{Ethical statement}

The study was approved by the Committees of Ethics in Animal Experimentation at the Universidade Federal de Ouro Preto (protocol no. 083/2007), Universidade Federal de Minas Gerais (protocol no. 020/2007), and of the City Council of Belo Horizonte (protocol no. 001/2008). All procedures were conducted according to the guidelines set by the Brazilian Animal Experimental Collage (COBEA), Federal Law number 11794. Owners of the dogs participating in the project were informed of the research objectives and signed the Informed Consent Form before sample collection. 


\section{Results}

To check for the influence of gender and age of the animals on the results we evaluated the homogeneity of the clinical groups. It was observed that there is no significant difference between the groups regarding gender and age of animals (data not show).

\subsection{Hematological profile}

Evaluation of hematological parameters showed severe anemia in the SD group, with significant decreases $(p<0.0001)$ in the number of erythrocytes, hemoglobin and hematocrit. Decreased erythrocyte counts were observed in the $\mathrm{AD}-\mathrm{II}$ and $\mathrm{SD}$ groups compared with the $\mathrm{CD}$ and $\mathrm{AD}$ I groups. Furthermore, hemoglobin concentrations were reduced in the $\mathrm{AD}-\mathrm{II}$ group compared with the $\mathrm{CD}$ and $\mathrm{AD}-\mathrm{I}$ groups. In the SD group, hemoglobin and hematocrit were reduced compared with all other groups. The white blood cells analysis revealed reductions in leukocyte counts in the AD-II group compared with the AD-I group $(p=0.0052)$. Absolute values of eosinophils, monocytes and lymphocytes were decreased in the AD-II and SD groups compared with the $\mathrm{CD}$ and AD-I groups $(p<0.0001)$. Platelet counts were reduced $(p=0.0098)$ in the SD group compared with the AD-I dogs (Table 1 ).

\subsection{Immunophenotyping of circulating $T$ lymphocytes and their subsets, $B$ lymphocytes and monocytes}

Animals from the AD-II and SD groups showed significantly decreased absolute values of $\mathrm{T}$ lymphocytes $\left(\mathrm{CD}^{+}\right)$ and their subpopulations $\left(\mathrm{CD} 4^{+}\right.$and $\left.\mathrm{CD}^{+}\right)$compared with the CD and AD-I groups $(p<0.0001)$. A negative correlation was observed with the clinical status. The $\mathrm{CD} 4^{+} / \mathrm{CD}^{+}$ $T$ lymphocyte ratio was significantly higher in the AD-I and SD groups compared with the $\mathrm{CD}$ and $\mathrm{AD}$-II groups (Fig. 1). In addition, the $\mathrm{AD}-\mathrm{II}$ and $\mathrm{SD}$ dogs had lower B-lymphocyte counts $\left(\mathrm{CD} 21^{+}\right)$than the $\mathrm{CD}$ and $\mathrm{AD}-\mathrm{I}$ groups $(p<0.0001)$. No difference was observed in monocytes compared with all groups. A negative correlation was also found between the number of B lymphocytes, monocytes and clinical groups (Fig. 2).

\subsection{Intracytoplasmic synthesis of IFN- $\gamma^{+}$and IL-4 $4^{+}$by} eosinophils and neutrophils, after in vitro antigen-specific stimulation

After stimulation with SLAi, neutrophils had higher frequency of IFN- $\gamma^{+}$in the AD-II and SD groups in comparison with the CD and AD-I groups. Synthesis of IL- $4^{+}$ was reduced in the AD-I group compared with all other groups $(p<0.0001)$. In addition, a positive correlation between clinical ongoing CVL and IFN- $\gamma^{+}$or $\mathrm{IL}^{+}$neutrophils was observed, and the IFN- $\gamma^{+} / \mathrm{IL}-4^{+}$ratio was increased in the AD-II and SD groups compared with the $C D$ group ( $p=0.0002$ ). However, no significant changes were observed in cytokine-producing eosinophils after in vitro SLAi stimulation (Fig. 3).

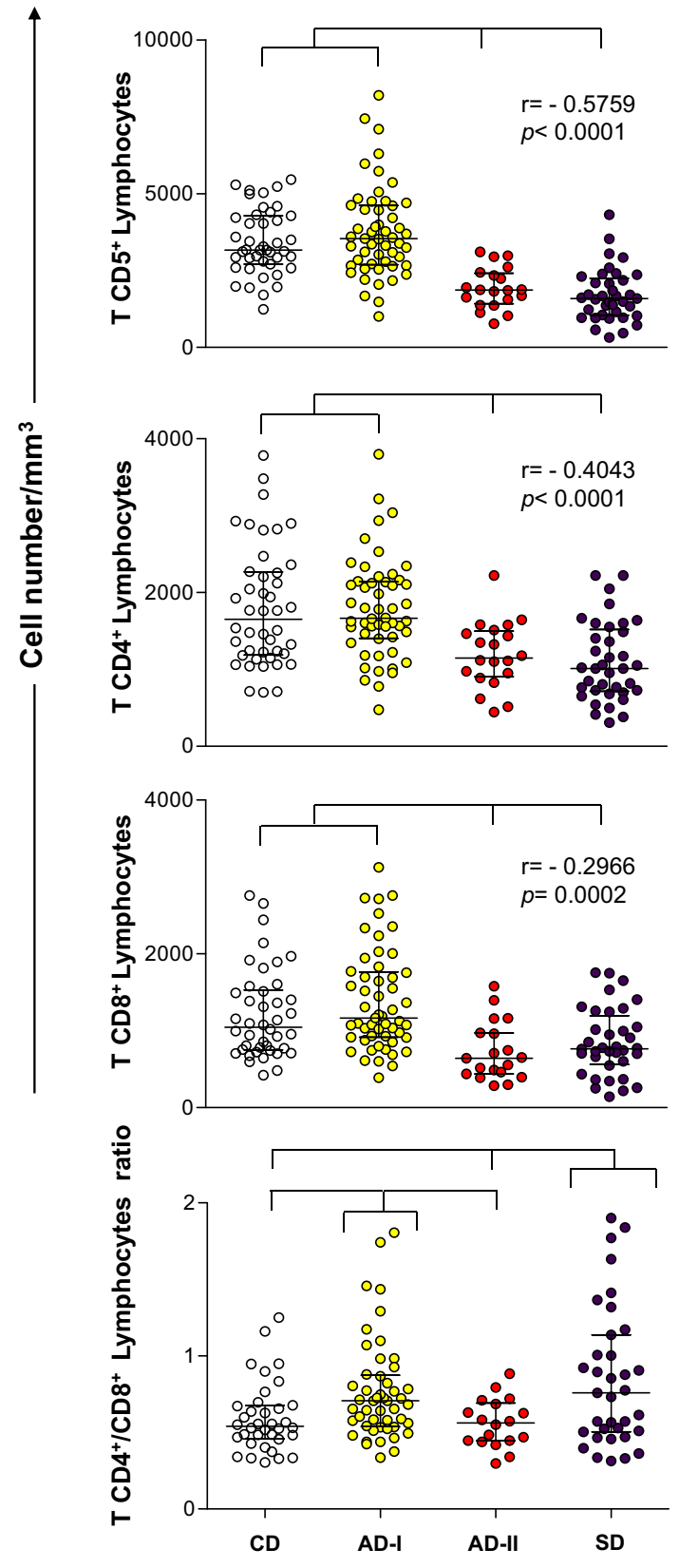

Clinical groups

Fig. 1. Immunophenotypic profile of lymphocytes in peripheral blood of dogs naturally infected by $L$. infantum categorized by their clinical status and laboratory results as asymptomatic-I (AD-I), asymptomatic-II (ADII) and symptomatic (SD). Noninfected dogs were used as controls (CD). Results are expressed as absolute cell counts in scatter plots, and bars represent median and interquartile range. Significant differences $(p<0.05)$ are indicated by connecting lines between the groups. The Pearson correlation $(r)$ and $p$ value shown in the graphics demonstrate correlations between $\mathrm{T} \mathrm{CD}^{+}$lymphocytes and the T subsets $\left(\mathrm{CD} 4^{+}\right.$and $\left.\mathrm{CD} 8^{+}\right)$with clinical progression. 


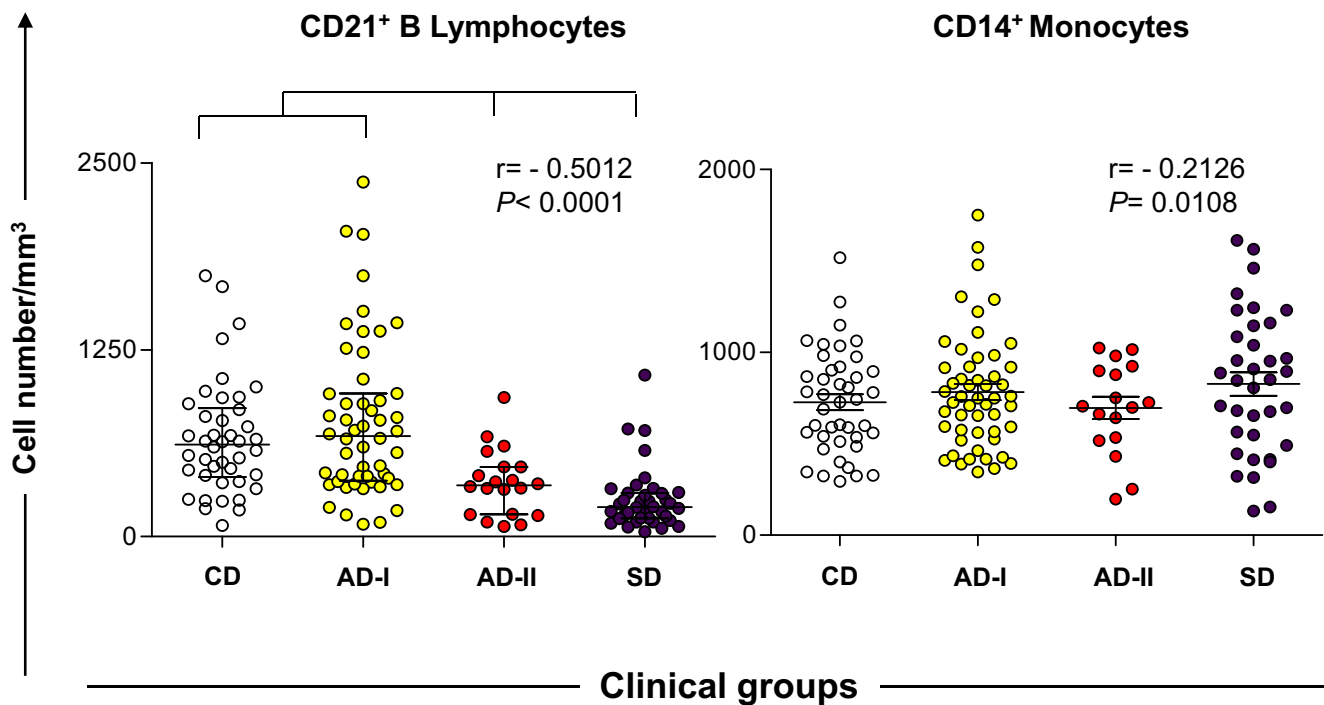

Fig. 2. Immunophenotypic profile of B lymphocytes and monocytes in peripheral blood of dogs naturally infected by $L$. infantum categorized by their clinical status and laboratory results as asymptomatic-I (AD-I), asymptomatic-II (AD-II) and symptomatic (SD). Noninfected dogs were used as controls (CD). Results are expressed as absolute cell counts in scatter plots, and bars represent median and interquartile range. Significant differences $(p<0.05)$ are indicated by connecting lines between the groups. The Pearson correlation $(r)$ at $p<0.05$ showed correlation between production of $\mathrm{CD} 21^{+} \mathrm{B}$ lymphocytes and $\mathrm{CD} 14^{+}$monocytes with clinical progression.

3.4. Intracytoplasmic synthesis of IFN- $\gamma^{+}$and IL-4+ by lymphocyte subsets $\left(C D 4^{+}\right.$and $\left.C D 8^{+}\right)$after in vitro antigen-specific stimulation

Increased percentages of IFN- $\gamma$ producing $\mathrm{CD} 4^{+}$or $\mathrm{CD} 8^{+}$ lymphocyte subsets were observed in the AD-II and SD groups compared with the CD and AD-I groups $(p<0.0001)$. Moreover, we observed an increased percentage of IL- $4^{+}$ $\mathrm{CD}^{+}$lymphocytes in the SD group as compared with the CD group $(p=0.0005)$. The percentage of IL- $4^{+} \mathrm{CD} 8^{+}$lymphocytes was higher in the AD-II and SD groups compared with the $\mathrm{CD}$ and $\mathrm{AD}-\mathrm{I}$ groups, and a positive correlation was found between the synthesis of IFN- $\gamma^{+}$or IL- $4^{+}$by these cells and the clinical status $(p<0.0001)$. In an analysis of the IFN- $\gamma^{+} / \mathrm{IL}-4^{+} \mathrm{CD} 4^{+}$or $\mathrm{CD} 8^{+}$cell ratio, no difference was observed in the experimental groups (Fig. 4).

\section{Discussion}

Studies of the hematological and immunological profiles of dogs naturally infected by $L$. infantum and presenting different clinical profiles are important in assessing biomarkers related to the pathogenesis and prognosis of CVL (Reis et al., 2009). The search for biomarkers may be important to better predict the evolution of canine disease, since the progression of infection depends on the efficiency of the immune response of the host. Thus, the identification and characterization of biomarkers can be important in the development of diagnosis/prognosis tests, vaccines and therapies evaluations applied to CVL.

In the present study, disease progression was associated with changes in various hematological parameters such as significant reduction of erythrocytes, hemoglobin and hematocrit, which characterized severe anemia, especially in symptomatic dogs. Our findings corroborate previous studies that identified anemia as a common hematological event in active and severe CVL (Reis et al., 2006a; da Costa-Val et al., 2007; de Freitas et al., 2012). De Luna et al. (2000) suggested that anemia would result in impaired erythrocyte membrane fluidity in CVL, which would favor mechanical sequestration of erythrocytes into the spleen and/or alter receptor-ligand erythrocyte cytoadherence mechanisms. In addition to anemia, seropositive dogs (AD-II and SD groups) presented eosinopenia, lymphopenia and monocytopenia as previously documented by Reis et al. (2006b). It has been shown that reduced white blood cell counts in symptomatic dogs may be associated with bone marrow dysfunction, with intense parasitism causing decreased hematopoiesis and redirecting bone marrow function (Tropia de Abreu et al., 2011). Nevertheless, considering that the dogs are from endemic area, the hematologic changes can not be entirely attributed to the CVL, since other canine vector-borne diseases that present similar laboratory findings were not ruled out.

In the present study, a distinct profile of total Tcirculating lymphocytes was observed among seronegative and seropositive animals. Considering the T-cell immune response found in the AD-I group (normal counts), a possible hypothesis is that this group presented a resistance profile. Other possibilities such as infection at the initial stage, when parasite load is minimal and dispersion reduced may characterize this group of animals. On the other hand, outstandingly decreased levels of $\mathrm{CD}^{+}$ T-lymphocytes were found in seropositive dogs (AD-II and SD), and there was a high correlation with clinical evolution, which clearly indicated immunosuppression of circulating T-lymphocytes contributing to susceptibility to infection and disease progression. Researchers have previously reported a reduction in the total T-lymphocyte 


\section{Eosinophils}
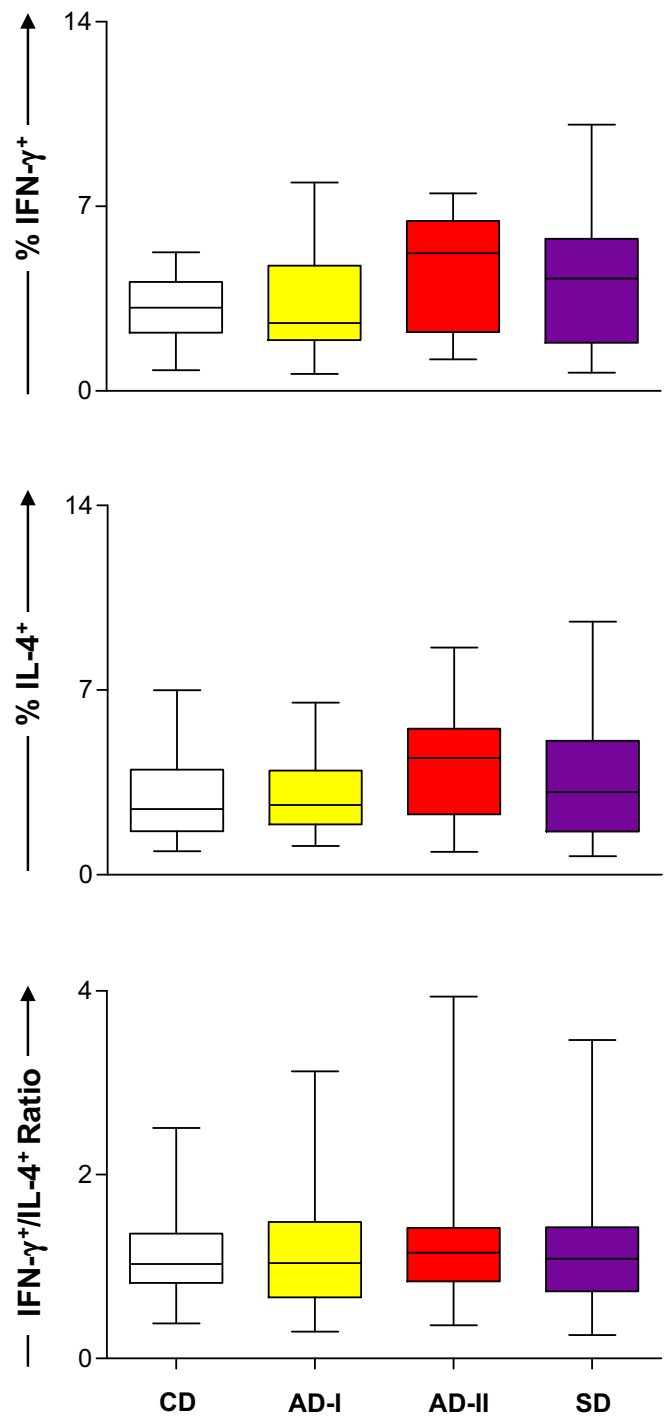

Neutrophils
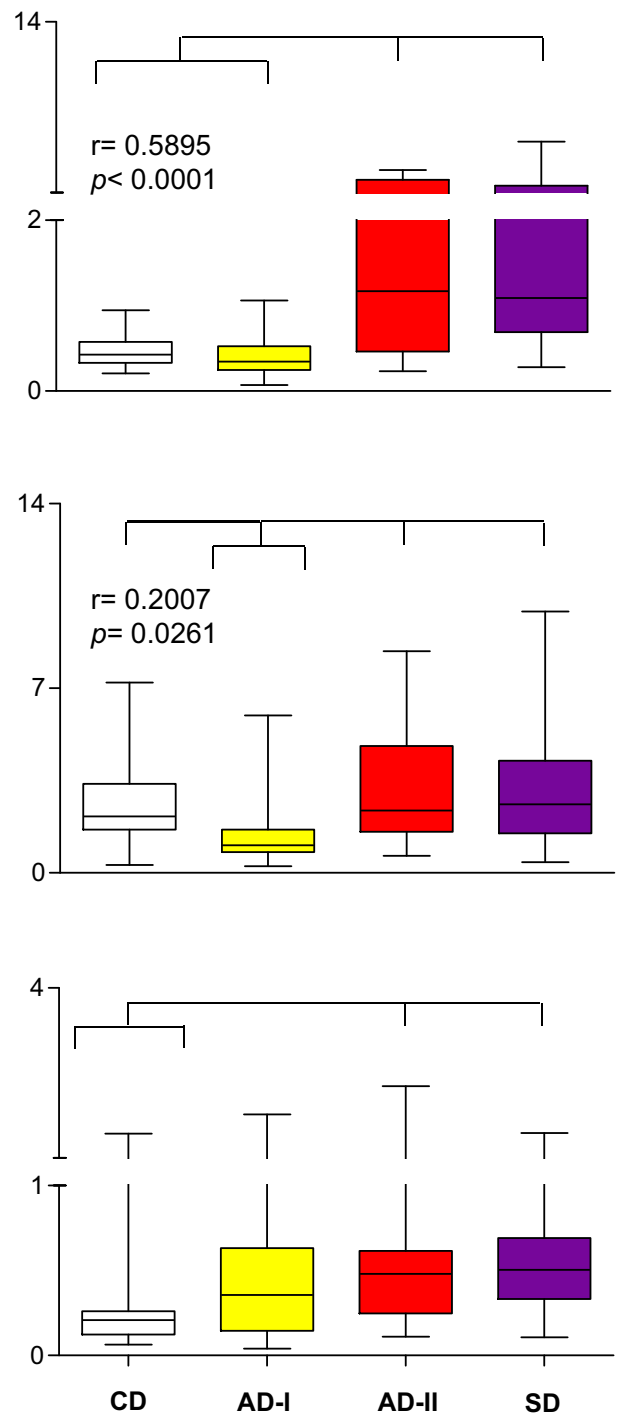

\section{Clinical groups}

Fig. 3. Percentage of neutrophils and eosinophils producers of IFN- $\gamma^{+}$, IL- $4^{+}$and ratio IFN- $\gamma^{+} / \mathrm{IL}-4^{+}$in stimulated culture (SLAi) of dogs naturally infected by L. infantum categorized by their clinical status and laboratory results as asymptomatic-I (AD-I), asymptomatic-II (AD-II) and symptomatic (SD). Noninfected dogs were used as controls (CD). Bars represent minimum and maximum values; boxes display median and interquartile range. Significant differences $(p<0.05)$ are indicated by connecting lines. The Pearson correlation $(r)$ and $p$ value show correlation between a production of IFN- $\gamma^{+}$and IL- $4^{+}$by neutrophils with clinical progression.

population in symptomatic dogs (Bourdoiseau et al., 1997; Reis et al., 2006b; Alexandre-Pires et al., 2010), and this reduction was associated with uncontrolled parasitism in these animals.

The $\mathrm{CD}^{+}$and $\mathrm{CD}^{+}{ }^{+}$-cell subsets followed a similar profile as observed for the $\mathrm{CD} 5^{+} \mathrm{T}$ lymphocytes, with reductions in the AD-II and SD groups. Decreased frequency of the $\mathrm{CD}^{+} \mathrm{T}$-cell subpopulation in active CVL has been confirmed by many groups (Bourdoiseau et al., 1997; Moreno et al., 1999; Reis et al., 2006b; Guerra et al., 2009). This reduction found in seropositive animals appears to be a critical factor for parasite replication, indicating a poor prognosis of disease progression. Furthermore, $\mathrm{CD}^{+} \mathrm{T}$ cells have been implicated in the control of visceralizing species of Leishmania (Tsagozis et al., 2003). Some researchers have associated the level of these cells with protection during asymptomatic CVL (Pinelli et al., 1995; Reis et al., 2006b; Coura-Vital et al., 2011a) in dogs with low tissue parasitism (Guerra et al., 2009) and/or in LBSap-vaccinated dogs (Giunchetti et al., 2007). Thus, our results suggest that CD8 ${ }^{+}$ 
CD4+ lymphocytes
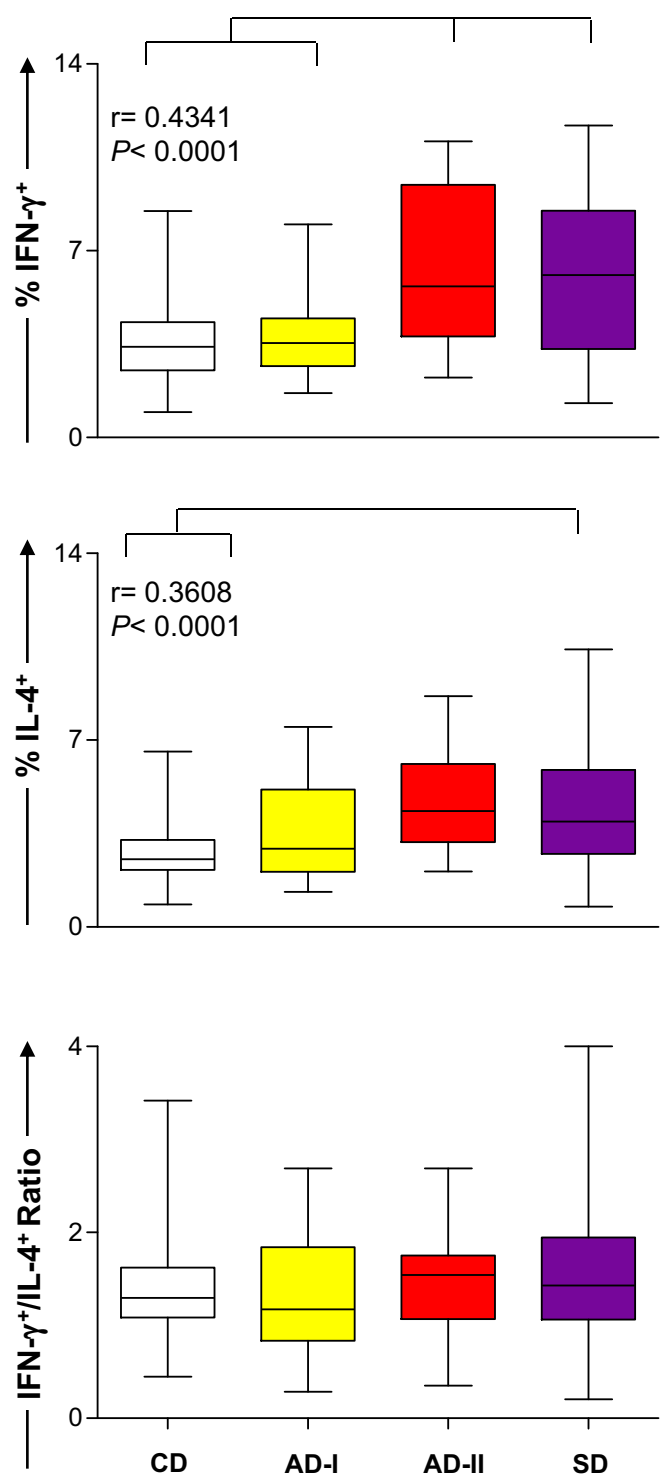

CD8+ lymphocytes
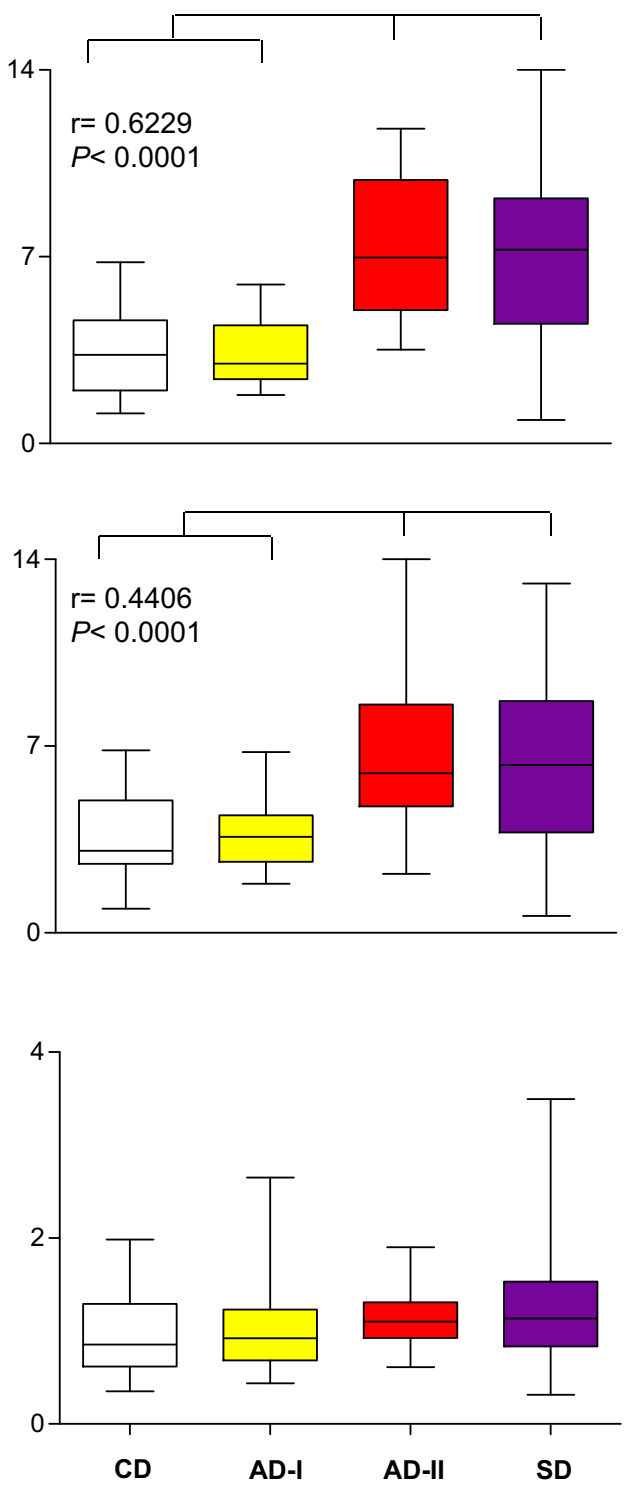

\section{Clinical groups}

Fig. 4. Percentage of $\mathrm{CD}^{+}$and $\mathrm{CD} 8^{+}$T-lymphocyte producers of IFN- $\gamma^{+}$, IL- $4^{+}$and the IFN- $\gamma^{+} / \mathrm{IL}-4^{+}$ratio in stimulated culture (SLAi) derived from dogs naturally infected by L. infantum categorized by their clinical status and laboratory results as asymptomatic-I (AD-I), asymptomatic-II (AD-II) and symptomatic (SD). Noninfected dogs were used as controls (CD). Bars represent minimum and maximum values; boxes display median and interquartile range. Significant differences $(p<0.05)$ are indicated by connecting lines. The Pearson correlation $(r)$ and $p$ value show correlation between a production of IFN- $\gamma^{+}$ and IL- $4^{+}$by $\mathrm{CD} 4^{+}$and $\mathrm{CD} 8^{+} \mathrm{T}$ lymphocytes with clinical progression.

T cells mediate the immune response by cytotoxic mechanisms that assist in protection during the early phase of the infections as observed in the AD-I group. However, reduced levels of this cell type in the AD-II group cannot control the infection, indicating a poor prognosis as observed in the SD group. Therefore, the evaluation of T-cell subsets $\left(C D 4^{+}\right.$ and $\mathrm{CD}^{+}$) represents an important biomarker of clinical progression in CVL.
Interestingly, we observed a decrease in the population of B lymphocytes $\left(\mathrm{CD} 21^{+}\right)$in both the AD-II and SD groups. Regarding the natural history of CVL, Reis et al. (2006b) reported that the decrease in CD $21^{+}$B cells could be related to increased parasite load in the bone marrow and the severity of CVL clinical signs. Other researchers have reported similar results, especially in symptomatic dogs, which suggests that the decrease of $\mathrm{CD} 21^{+} \mathrm{B}$ lymphocytes 
may be a biomarker for susceptibility and/or severe CVL (Bourdoiseau et al., 1997; Coura-Vital et al., 2011a).

Recent studies showed that neutrophils play an important regulatory role in the early stages of Leishmania infection (Peters and Sacks, 2009; Ritter et al., 2009). Studies have also shown that these cells have a direct impact on the death of the parasite and the development of a protective immune response against infections caused by $L$. donovani and L. infantum (Rousseau et al., 2001; McFarlane et al., 2008). Thus, these cells could provide an important link between innate and adaptive immunity during parasite infection, and they are on of them responsible for the visceralization of the parasitism for many lymphoid organs (Carvalho et al., 2012). In this study, it was observed for the first time that canine blood neutrophils, when stimulated in vitro with SLAi, were able to produce high levels of IFN- $\gamma^{+}$in AD-II and SD animals compared with $\mathrm{CD}$ and $\mathrm{AD}-\mathrm{I}$ dogs. These findings were confirmed by the correlation observed when dogs exhibit active CVL. In addition, high levels of IFN- $\gamma^{+}$neutrophils are an attempt by the immune system to decrease the parasite load, but IFN- $\gamma$ alone cannot protect the animals during infection (Chamizo et al., 2005; Lage et al., 2007; Reis et al., 2010). Interestingly, the AD-I group showed reduced synthesis of IL- 4 by neutrophils compared with the other groups. In human patients with active VL, increased synthesis of IL-4 by neutrophils stimulated by soluble Leishmania antigens has been reported (Peruhype-Magalhaes et al., 2005). IL-4 appears to be related to the severity of CVL (Quinnell et al., 2001), thus this cytokine could be an important biomarker of susceptibility in canines naturally infected by L. infantum. Although Peruhype-Magalhaes et al. (2005) found a high frequency of stimulated eosinophil IFN $-\gamma^{+}$in human VL, we did not observe this finding either for neutrophil or eosinophil IL- $4^{+}$in dogs naturally infected by L. infantum.

An evaluation of intracytoplasmic cytokine expression in in vitro experiments with T-cell subsets $\left(\mathrm{CD} 4^{+}\right.$and $\mathrm{CD} 8^{+}$ lymphocytes) showed that both subsets produced high levels of IFN- $\gamma$ (mainly by CD8 ${ }^{+}$lymphocytes with high correlation) in seropositive animals (AD-II and SD groups) in comparison with the $\mathrm{CD}$ and AD-I groups. Previous studies have reported that high expression of IFN- $\gamma$ is associated with symptomatic disease (Lage et al., 2007; RodriguezCortes et al., 2007; Costa et al., 2013). However, Carrillo et al. (2007) found reduced expression of IFN- $\gamma$ in experimentally infected dogs presenting symptomatic VL. These high levels of IFN- $\gamma$ produced by lymphocytes in AD-II and SD dogs indicate an attempt to control infection in these animals. However, our results also suggested that IFN- $\gamma$ was not sufficient to prevent disease, and it could not be considered as a marker of resistance on these animals. Perhaps, successful infection control could not achieved because there was a balance with modulating cytokines such as IL-10 and TGF- $\beta$ that undermined clinical improvement in animals. Additional studies evaluating the profile of cytokine modulators will be important to clarify this fact.

Regarding the participation of IL-4, many researchers demonstrated that this cytokine is associated with susceptibility to disease and is associated with an increase in parasitic load in CVL (Pinelli et al., 1999b; Quinnell et al., 2001; Alves et al., 2009). Strauss-Ayali et al. (2007) reported that early expression of IL-4 measured in spleen cells plays an important role in the persistence of parasites despite high expression of IFN- $\gamma$ (Strauss-Ayali et al., 2007). Based on these findings we suggest that AD-I animals have extremely low parasitism, with little stimulation of the IL- $4^{+}$synthesis by lymphocytes, which may be a protective factor for these animals. In contrast, the production of $\mathrm{IL}-4^{+}$by $\mathrm{CD}^{+}$and $\mathrm{CD} 8^{+}$cells detected in seropositive dogs (AD-II and SD) may contribute to better understand the persistence and parasite replication observed in severe CVL, as previously documented by Guerra et al. (2009).

In conclusion, this study showed for the first time that the AD-I group does not differ from healthy animals in that no significant alteration occurs in the cell population evaluated and there is no activation of the Type 2 immune response, which effectively confers a resistance profile for them. In contrast, the animals from the AD-II and SD groups exhibited a mixed immune profile (Type 1 and 2 ) in parallel with the immunosuppression that affected both the $\mathrm{T}$ and $B$ compartments, with a concomitant presence of outstanding IFN $-\gamma^{+}$and IL- $4^{+}$produced by neutrophils and T lymphocytes, making them unable to control parasite replication.

\section{Acknowledgements}

This study was supported by the following grants: Federal University of Ouro Preto; DECIT/MS/CNPq/BR/grant: 576062/2008-1; FAPEMIG/BR/grant: CBB-APQ-3073-4.01/ 07, CNPq/BR/grant: 472554/2007-7; PPSUS/MS/CNPq/ FAPEMIG/SES-MG/grant CBB-APQ-00356-10; FAPEMIG/ PPM and PNPD/Institutional/2011. The funders had no role in study design, data collection or analysis, the decision to publish, or the preparation of the manuscript. ABR, CMC, RCG, ATC, OAMF, and MC are grateful for CNPq fellowships, WCV is grateful to the PNPD/CAPES fellowships and Federal University of Ouro Preto. We also thank the staff of the Secretaria Municipal de Saúde de Belo Horizonte, Minas Gerais, for cooperation, logistical support, and special dedication to this work.

\section{References}

Alexandre-Pires, G., de Brito, M.T., Alguero, C., Martins, C., Rodrigues, O.R., da Fonseca, I.P., Santos-Gomes, G., 2010. Canine leishmaniasis. Immunophenotypic profile of leukocytes in different compartments of symptomatic, asymptomatic and treated dogs. Vet. Immunol. Immunopathol. 137, 275-283.

Alves, C.F., de Amorim, I.F., Moura, E.P., Ribeiro, R.R., Michalick, M.S., Kalapothakis, E., Bruna-Romero, O., Tafuri, W.L., Teixeira, M.M., Melo, M.N., 2009. Expression of IFN-gamma, TNF-alpha, IL-10 and TGF-beta in lymph nodes associates with parasite load and clinical form of disease in dogs naturally infected with Leishmania (Leishmania) chagasi. Vet. Immunol. Immunopathol. 128, 349-358.

Bourdoiseau, G., Bonnefont, C., Magnol, J.P., Saint-Andre, I., Chabanne, L., 1997. Lymphocyte subset abnormalities in canine leishmaniasis. Vet. Immunol. Immunopathol. 56, 345-351

Carrillo, E., Ahmed, S., Goldsmith-Pestana, K., Nieto, J., Osorio, Y., Travi, B., Moreno, J., McMahon-Pratt, D., 2007. Immunogenicity of the P8 amastigote antigen in the experimental model of canine visceral leishmaniasis. Vaccine 25, 1534-1543.

Carrillo, E., Moreno, J., 2009. Cytokine profiles in canine visceral leishmaniasis. Vet. Immunol. Immunopathol. 128, 67-70.

Carvalho, L.P., Petritus, P.M., Trochtenberg, A.L., Zaph, C., Hill, D.A., Artis, D., Scott, P., 2012. Lymph node hypertrophy following Leishmania major infection is dependent on TLR9. J. Immunol. 188, 1394-1401. 
Chamizo, C., Moreno, J., Alvar, J., 2005. Semi-quantitative analysis of cytokine expression in asymptomatic canine leishmaniasis. Vet. Immunol. Immunopathol. 103, 67-75.

Correa, A.P., Dossi, A.C., de Oliveira Vasconcelos, R., Munari, D.P., de Lima, V.M., 2007. Evaluation of transformation growth factor beta1, interleukin-10, and interferon-gamma in male symptomatic and asymptomatic dogs naturally infected by Leishmania (Leishmania) chagasi. Vet. Parasitol. 143, 267-274.

Costa, D.J., Carvalho, R.M., Abbehusen, M., Teixeira, C., Pitombo, M., Trigo, J., Nascimento, F., Amorim, L., Abreu-Silva, A.L., do Socorro Pires Cruz, M., Miranda, J.C., Fukutani, K., de Oliveira, C.I., Barral, A., Barral-Netto, M., Brodskyn, C., 2013. Experimental infection of dogs with leishmania and saliva as a model to study canine visceral leishmaniasis. PLOS ONE 8, e60535.

Coura-Vital, W., Marques, M.J., Giunchetti, R.C., Teixeira-Carvalho, A., Moreira, N.D., Vitoriano-Souza, J., Vieira, P.M., Carneiro, C.M., CorreaOliveira, R., Martins-Filho, O.A., Carneiro, M., Reis, A.B., 2011 a. Humoral and cellular immune responses in dogs with inapparent natural Leishmania infantum infection. Vet. J. 190, e43-e47.

Coura-Vital, W., Marques, M.J., Veloso, V.M., Roatt, B.M., Aguiar-Soares, R.D., Reis, L.E., Braga, S.L., Morais, M.H., Reis, A.B., Carneiro, M., 2011 b. Prevalence and factors associated with Leishmania infantum infection of dogs from an urban area of Brazil as identified by molecular methods. PLoS Negl. Trop. Dis. 5, e1291.

Coura-Vital, W., Reis, A.B., Fausto, M.A., Leal, G.G.A., Marques, M.J., Veloso, V.M., Carneiro, M., 2013. Risk factors for seroconversion by Leishmania infantum in a cohort of dogs from an endemic area of Brazil. PLOS ONE 8, e71833.

da Costa-Val, A.P., Cavalcanti, R.R., de Figueiredo Gontijo, N., Michalick, M.S., Alexander, B., Williams, P., Melo, M.N., 2007. Canine visceral leishmaniasis: relationships between clinical status, humoral immune response, haematology and Lutzomyia(Lutzomyia) longipalpis infectivity. Vet. J. 174, 636-643.

de Freitas, J.C., Lopes-Neto, B.E., de Abreu, C.R., Coura-Vital, W., Braga, S.L., Reis, A.B., Nunes-Pinheiro, D.C., 2012. Profile of anti-Leishmania antibodies related to clinical picture in canine visceral leishmaniasis. Res. Vet. Sci. 93, 705-709.

De Luna, R., Ferrante, M., Severino, L., Ambrosio, R., Piantedosi, D., Gradoni, L., Lucisano, A., Persechino, A., 2000. Decreased lipid fluidity of the erythrocyte membrane in dogs with leishmaniasis-associated anaemia. J. Comp. Pathol. 122, 213-216

Degrave, W., Fernandes, O., Campbell, D., Bozza, M., Lopes, U., 1994. Use of molecular probes and PCR for detection and typing of Leishmania-a mini-review. Mem. Inst. Oswaldo Cruz 89, 463-469.

Fraga, D.B., Solca, M.S., Silva, V.M., Borja, L.S., Nascimento, E.G., Oliveira, G.G., Pontes-de-Carvalho, L.C., Veras, P.S., Dos-Santos, W.L., 2012. Temporal distribution of positive results of tests for detecting Leishmania infection in stray dogs of an endemic area of visceral leishmaniasis in the Brazilian tropics: a 13 years survey and association with human disease. Vet. Parasitol. 190, 591-594.

Giunchetti, R.C., Correa-Oliveira, R., Martins-Filho, O.A., Teixeira-Carvalho, A., Roatt, B.M., de Oliveira Aguiar-Soares, R.D., de Souza, J.V., das Dores Moreira, N., Malaquias, L.C., Mota e Castro, L.L., de Lana, M., Reis, A.B., 2007. Immunogenicity of a killed Leishmania vaccine with saponin adjuvant in dogs. Vaccine 25, 7674-7686.

Giunchetti, R.C., Mayrink, W., Genaro, O., Carneiro, C.M., Correa-Oliveira, R., Martins-Filho, O.A., Marques, M.J., Tafuri, W.L., Reis, A.B., 2006. Relationship between canine visceral leishmaniosis and the Leishmania (Leishmania) chagasi burden in dermal inflammatory foci. J. Comp. Pathol. 135, 100-107.

Grimaldi Jr., G., Teva, A., Santos, C.B., Ferreira, A.L., Falqueto, A., 2012. The effect of removing potentially infectious dogs on the numbers of canine Leishmania infantum infections in an endemic area with high transmission rates. Am. J. Trop. Med. Hyg. 86, 966-971.

Guerra, L.L., Teixeira-Carvalho, A., Giunchetti, R.C., Martins-Filho, O.A., Reis, A.B., Correa-Oliveira, R., 2009. Evaluation of the influence of tissue parasite density on hematological and phenotypic cellular parameters of circulating leukocytes and splenocytes during ongoing canine visceral leishmaniasis. Parasitol. Res. 104, 611-622.

Lage, R.S., Oliveira, G.C., Busek, S.U., Guerra, L.L., Giunchetti, R.C., CorreaOliveira, R., Reis, A.B., 2007. Analysis of the cytokine profile in spleen cells from dogs naturally infected by Leishmania chagasi. Vet. Immunol. Immunopathol. 115, 135-145.

Lowry, O.H., Rosebrough, N.J., Farr, A.L., Randall, R.J., 1951. Protein measurement with the Folin phenol reagent. J. Biol. Chem. 193, 265-275.

McFarlane, E., Perez, C., Charmoy, M., Allenbach, C., Carter, K.C., Alexander, J., Tacchini-Cottier, F., 2008. Neutrophils contribute to development of a protective immune response during onset of infection with Leishmania donovani. Infect. Immun. 76, 532-541.
Menezes-Souza, D., Correa-Oliveira, R., Guerra-Sa, R., Giunchetti, R.C., Teixeira-Carvalho, A., Martins-Filho, O.A., Oliveira, G.C., Reis, A.B., 2011. Cytokine and transcription factor profiles in the skin of dogs naturally infected by Leishmania (Leishmania) chagasi presenting distinct cutaneous parasite density and clinical status. Vet. Parasitol. 177, 39-49

Moreno, J., Alvar, J., 2002. Canine leishmaniasis: epidemiological risk and the experimental model. Trends Parasitol. 18, 399-405.

Moreno, J., Nieto, J., Chamizo, C., Gonzalez, F., Blanco, F., Barker, D.C., Alva, J., 1999. The immune response and PBMC subsets in canine visceral leishmaniasis before, and after, chemotherapy. Vet. Immunol. Immunopathol. 71, 181-195.

Passos, V.M., Fernandes, O., Lacerda, P.A., Volpini, A.C., Gontijo, C.M., Degrave, W., Romanha, A.J., 1999. Leishmania (Viannia) braziliensis is the predominant species infecting patients with American cutaneous leishmaniasis in the State of Minas Gerais, Southeast Brazil. Acta Trop. $72,251-258$

Peruhype-Magalhaes, V., Martins-Filho, O.A., Prata, A., Silva, L.A., Rabello, A., Teixeira-Carvalho, A., Figueiredo, R.M., Guimaraes-Carvalho, S.F., Ferrari, T.C., Correa-Oliveira, R., 2005. Immune response in human visceral leishmaniasis: analysis of the correlation between innate immunity cytokine profile and disease outcome. Scand. J. Immunol. 62, 487-495.

Peters, N.C., Sacks, D.L., 2009. The impact of vector-mediated neutrophil recruitment on cutaneous leishmaniasis. Cell Microbiol. 11, 1290-1296.

Pinelli, E., Gonzalo, R.M., Boog, C.J., Rutten, V.P., Gebhard, D., del Real, G., Ruitenberg, E.J., 1995. Leishmania infantum-specific T cell lines derived from asymptomatic dogs that lyse infected macrophages in a major histocompatibility complex-restricted manner. Eur. J. Immunol. 25, 1594-1600.

Pinelli, E., Killick-Kendrick, R., Wagenaar, J., Bernadina, W., del Real, G., Ruitenberg, J., 1994. Cellular and humoral immune responses in dogs experimentally and naturally infected with Leishmania infantum. Infect. Immun. 62, 229-235.

Pinelli, E., van der Kaaij, S.Y., Broeren, C.P., Ruitenberg, E.J., Rutten, V.P., 1999a. Measurement of dog cytokines by reverse transcriptionquantitative competitive polymerase chain reaction. Immunogenetics 49, 696-699.

Pinelli, E., Van der Kaaij, S.Y., Slappendel, R., Fragio, C., Ruitenberg, E.J., Bernadina, W., Rutten, V.P., 1999b. Detection of canine cytokine gene expression by reverse transcription-polymerase chain reaction. Vet. Immunol. Immunopathol. 69, 121-126.

Quinnell, R.J., Courtenay, O., Shaw, M.A., Day, M.J., Garcez, L.M., Dye, C., Kaye, P.M., 2001. Tissue cytokine responses in canine visceral leishmaniasis. J. Infect. Dis. 183, 1421-1424.

Reis, A.B., Giunchetti, R.C., Carrillo, E., Martins-Filho, O.A., Moreno, J., 2010. Immunity to Leishmania and the rational search for vaccines against canine leishmaniasis. Trends Parasitol. 26, 341-349.

Reis, A.B., Martins-Filho, O.A., Teixeira-Carvalho, A., Carvalho, M.G. Mayrink, W., Franca-Silva, J.C., Giunchetti, R.C., Genaro, O., CorreaOliveira, R., 2006a. Parasite density and impaired biochemical/hematological status are associated with severe clinical aspects of canine visceral leishmaniasis. Res. Vet. Sci. 81, 68-75.

Reis, A.B., Martins-Filho, O.A., Teixeira-Carvalho, A., Giunchetti, R.C., Carneiro, C.M., Mayrink, W., Tafuri, W.L., Correa-Oliveira, R., 2009. Systemic and compartmentalized immune response in canine visceral leishmaniasis. Vet. Immunol. Immunopathol. 128, 87-95.

Reis, A.B., Teixeira-Carvalho, A., Giunchetti, R.C., Guerra, L.L., Carvalho, M.G., Mayrink, W., Genaro, O., Correa-Oliveira, R., Martins-Filho, O.A., 2006b. Phenotypic features of circulating leucocytes as immunological markers for clinical status and bone marrow parasite density in dogs naturally infected by Leishmania chagasi. Clin. Exp. Immunol. 146, 303-311.

Reis, A.B., Teixeira-Carvalho, A., Vale, A.M., Marques, M.J., Giunchetti, R.C., Mayrink, W., Guerra, L.L., Andrade, R.A., Correa-Oliveira, R., MartinsFilho, O.A., 2006c. Isotype patterns of immunoglobulins: hallmarks for clinical status and tissue parasite density in Brazilian dogs naturally infected by Leishmania (Leishmania) chagasi. Vet. Immunol. Immunopathol. 112, 102-116.

Ritter, U., Frischknecht, F., van Zandbergen, G., 2009. Are neutrophils important host cells for Leishmania parasites? Trends Parasitol. 25, 505-510.

Rodriguez-Cortes, A., Fernandez-Bellon, H., Ramis, A., Ferrer, L., Alberola, J., Solano-Gallego, L., 2007. Leishmania-specific isotype levels and their relationship with specific cell-mediated immunity parameters in canine leishmaniasis. Vet. Immunol. Immunopathol. 116, $190-198$.

Rousseau, D., Demartino, S., Ferrua, B., Michiels, J.F., Anjuere, F., Fragaki, K., Le Fichoux, Y., Kubar, J., 2001. In vivo involvement of 
polymorphonuclear neutrophils in Leishmania infantum infection. BMC Microbiol. 1, 17.

Strauss-Ayali, D., Baneth, G., Jaffe, C.L., 2007. Splenic immune responses during canine visceral leishmaniasis. Vet. Res. 38, 547-564.

Tropia de Abreu, R., Carvalho, M.G., Carneiro, C.M., Giunchetti, R.C., Teixeira-Carvalho, A., Martins-Filho, O.A., Coura-Vital, W., CorreaOliveira, R., Reis, A.B., 2011. Influence of clinical status and parasite load on erythropoiesis and leucopoiesis in dogs naturally infected with Leishmania (Leishmania) chagasi. PLoS ONE 6, e18873.

Tsagozis, P., Karagouni, E., Dotsika, E., 2003. CD8(+) T cells with parasitespecific cytotoxic activity and a Tc1 profile of cytokine and chemokine secretion develop in experimental visceral leishmaniasis. Parasite Immunol. 25, 569-579.

Volpini, A.C., Passos, V.M., Oliveira, G.C., Romanha, A.J., 2004. PCR-RFLP to identify Leishmania (Viannia) braziliensis and L. (Leishmania) amazonensis causing American cutaneous leishmaniasis. Acta Trop. 90, 31-37.

World Health Organization, 2010. Working to Overcome the Global Impact of Neglected Tropical Diseases: First WHO Report on Neglected Tropical Diseases. World Health Organization, Geneva. 